\title{
JURISPRUDENCIA AMBIENTAL EN ANDALUCÍA (SEGUNDO SEMESTRE 2017)
}

\author{
LORENZO MELLADO RUIZ \\ Profesor Titular de Derecho Administrativo \\ Universidad de Almería
}


Sumario. 1. Ejecución no autorizada de obras e instalaciones en el dominio público marítimoterrestre y ausencia de culpabilidad por incomplitud del título formal de compraventa; 2 . Inicio del cómputo del plazo para la interposición del recurso contencioso-administrativo contra actos locales e integración sustantiva del concepto de utilidad pública o interés social para la aprobación de proyectos de actuación; 3. Existencia de "continuidad ecológica" entre los ecosistemas marino y terrestre a efectos de su distribución competencial: contradicción de informes técnicos, mejor evidencia científica y carga de la prueba; 4 . Indefensión formal vs. indefensión material en los procedimientos de declaración de Zonas de Especial Conservación; 5. Daños por inundaciones y concurrencia pública de culpas; 6 . La modificación de documentos gráficos urbanísticos no es en cualquier caso una mera rectificación de errores; 7 . Plazo de aprobación del deslinde del dominio público hidráulico y requisitos para su ampliación excepcional; 8. Virtualidad anulatoria de la ausencia de los informes técnicos y de suficiencia de recursos hídricos en los procedimientos de declaración de interés turístico de proyectos de campos de golf: declaración "por silencio" e inmanencia de "lesividad" de la contradicción con la normativa ambiental reguladora

Por necesidades de ajuste temporal, sincronización y continuidad con la anterior crónica y accesibilidad a la propia jurisprudencia*, la presente crónica abarca el período comprendido entre febrero de 2017 y julio de 2017.

\section{Ejecución no autorizada de obras e instalaciones en el dominio público} marítimo-terrestre y ausencia de culpabilidad por incomplitud del título formal de compraventa

\section{STSJA de 27 de febrero de 2017 , rec. $1294 / 2012$}

La Sentencia del TSJA (Sala de lo Contencioso-Administrativo, Sección 1a, Sede de Granada) de 27 de febrero de 2017, rec. 1294/2012, resuelve el recurso contra la sanción impuesta por la realización de obras consistentes en el arreglo de un invernadero cambiando los palos por hierro galvanizado y renovando el alambrado y el plástico, ocupando parcialmente zona de dominio público marítimo-terrestre y de servidumbre de tránsito, sin la autorización pertinente.

En principio, según el art. 91.2.b) LC, la "ejecución no autorizada de obras e instalaciones en el dominio público marítimo-terrestre, así como el aumento de superficie, volumen o altura construidos sobre los autorizados" constituye infracción grave. Y aunque constan estos elementos típicos (invasión demanial y ausencia de autorización), se cuestiona a continuación el TSJA la existencia o no de culpabilidad adicional, concluyéndose que "no resulta suficientemente probada dicha culpabilidad, $y$, en concreto, la existencia de un nexo entre los elementos objetivo y subjetivo del injusto de la que se deduce la intencionalidad

\footnotetext{
*Fuente: CENDOJ
} 
del imputado" (F. J. $3^{\circ}$ ). La razón es que existiría un error de hecho propiciado por lo consignado en la escritura pública de compraventa de los terrenos a la Dirección General del Patrimonio del Estado, donde no se mencionaba precisamente la ocupación del dominio público. Habría una divergencia entre la realidad (con probada ocupación parcial del demanio, en una finca rústica segregada y transmitida lindante con zona marítima por el sur) y la "legalidad formal", con falta de consignación expresa al respecto. Por ello, señala el TSJA que si para que pueda sancionarse una infracción, en el caso concreto la prevista en el art. 97.1.b) LC, es necesaria la concurrencia del elemento subjetivo del injusto añadida a la de la tipicidad, habida cuenta de que en este caso el recurrente actuó en la confianza legítima de que su finca no invadía siquiera parcialmente el dominio público, amparado por los datos contenidos en una escritura en la que precisamente consta como transmitente del terreno el propio Estado español, la imposición de la sanción resulta improcedente.

La solución puede ser "formalmente" correcta, pero no deja de suscitar ciertos interrogantes desde la perspectiva de la tutela efectiva -y por tanto materialdel ambiente natural. Se hace primar en el caso la inexistencia de constancia documental -aún en escritura pública- sobre la realidad fáctica de ocupación o invasión, acreditada además oportunamente a través de los correspondientes informes, y, la virtualidad de un documento o título privado de transmisión sobre la tutela pública preventiva del dominio público marítimo-terrestre.

\section{Inicio del cómputo del plazo para la interposición del recurso} contencioso-administrativo contra actos locales e integración sustantiva del concepto de utilidad pública o interés social para la aprobación de proyectos de actuación

\section{STSJA de 18 de mayo de 2017, rec. 527/2015}

Resuelve la STSJA (Sala de lo Contencioso-Administrativo, Sección 4ạ , Sede de Granada), de 18 de mayo de 2017, rec. 527/2015, un recurso relativo a un proyecto de actuación para la construcción de una nave de servicios y almacenamiento y logística por parte de una localidad jienense.

Aclara en primer lugar el TSJA que el inicio del cómputo del plazo de dos meses para la interposición del correspondiente recurso contencioso- 
administrativo no viene determinado por la fecha de recepción por la Junta de Andalucía del extracto de los actos y acuerdos que están obligadas a remitir las entidades locales en los diez días siguientes a su adopción (en este caso, la aprobación por el Pleno municipal del proyecto de actuación), a través de medios electrónicos, sino por la propia fecha de la remisión telemática. Pero además, y en contra del criterio del Juzgador de instancia, estima la Sala del TSJA, en sintonía con otras sentencias precedentes, que sí sería de aplicación supletoria el art. 135 LEC, que prevé la posibilidad de interponer el recurso jurisdiccional hasta las 15 horas del día siguiente al del vencimiento del plazo.

Resuelve así el TSJA sobre la admisibilidad temporal del recurso.

En cuanto al fondo del asunto, se recuerda que la naturaleza del suelo no urbanizable impone, como regla general conocida, la imposibilidad de construir, aunque admitiendo ciertas excepciones legales. Una, en relación con las construcciones características de las explotaciones agrarias, que por su adecuación al destino natural del suelo son plenamente admisibles en principio, y otra, que es la que interesa aquí, en relación con las construcciones e instalaciones vinculadas a la ejecución, mantenimiento y servicio de las obras públicas, donde podría encuadrarse la instalación discutida, que no se adecúa al destino natural del suelo (rústico) y cuya autorización requiere el correspondiente proyecto de actuación o plan especial.

Pues bien, se enfrenta el TSJA a la comprobación de la existencia o no de utilidad pública o interés social como parámetros de legitimación de la aprobación del proyecto de actuación, conceptos, como se sabe, de claro contenido jurídico "indeterminado", y, en su traslado a la posibilidad de actuaciones de transformación física en el suelo no urbanizable, de interpretación expresamente restrictiva. Como cualquier excepción a una norma general prohibitiva, que salvaguarda la naturaleza del suelo en su estado original, las autorizaciones han de ser interpretadas siempre en sentido restrictivo, y tras haber quedado perfectamente acreditados los condicionamientos que permiten autorizar esas construcciones en suelo no urbanizable. $Y$ esa necesaria interpretación restrictiva determina que la utilidad pública o el interés social no puedan identificarse, sin más, con cualquier actividad industrial, comercial o negocial, en general, de la que se derive la 
satisfacción de una necesidad de los ciudadanos, ya que la extensión de la excepción legal a todo este tipo de instalaciones o actividades que, claro está, suponen una mayor creación de empleo y riqueza, supondría la conversión de la excepción en la regla general. En este caso concreto planteado, se cifra como motivo de utilidad pública de la instalación la "finalidad generadora de empleos", pero entiende el TSJA que se trata de una dicción meramente genérica, insuficiente, pues, para acreditar la concurrencia del requisito legal de utilidad pública o interés social (F. J. 4ำ).

También se alude al criterio de oportunidad para la implantación de tales servicios y actividades ante la carencia de oferta de suelos - de uso terciario o industrial- para ello (en terrenos no urbanos y a precios competitivos), pero entiende el TSJA por el contrario que el propio Proyecto denota una clara incidencia territorial de la actividad, constatándose la incidencia supramunicipal de la actuación proyectada, lo que determinaría, finalmente, que el instrumento de autorización en suelo no urbano fuera un plan especial, y no el simple proyecto de actuación aprobado en su momento (por aplicación del art. 42.4 LOUA).

3. Existencia de "continuidad ecológica" entre los ecosistemas marino y terrestre a efectos de su distribución competencial: contradicción de informes técnicos, mejor evidencia científica y carga de la prueba

STSJA de 25 de mayo de 2017, rec. $22 / 2016$

Se plantea la STSJA (Sala de lo Contencioso-administrativo, Sección $3^{a}$, Sede de Sevilla) de 25 de mayo de 2017, rec. 22/2016, la cuestión central de si para la declaración y aprobación del plan de gestión de una determinada Zona Especial de Protección se ha justificado por la Administración autonómica la continuidad ecológica entre medio marino y medio terrestre, de acuerdo con la mejor evidencia científica disponible.

Como se recuerda, las competencias de las Comunidades Autónomas en materia de espacios naturales protegidos no alcanzan, por regla general, al mar territorial, si bien las circunstancias y características específicas del espacio a proteger pueden demandar, en ocasiones excepcionales, que el mismo se extienda en alguna medida sobre dicha zona, singularmente cuando "así venga 
exigido por la continuidad y la unidad de dicho espacio físico", fundamentando, pues, la excepcionalidad de las medidas de alteración del sistema competencial en la propia concepción holística o sistémica del ambiente natural y la inconveniencia, en ocasiones, de su fragmentación tuitiva. Por ello, si una Comunidad Autónoma pretende declarar y gestionar espacios naturales protegidos en el mar debe acreditar dicha continuidad ecológica. Y no parece suficiente, como sucede en el caso planteado, el informe técnico de parte emitido por un profesor universitario. Como dice el TSJA, aun siendo su opinión lógicamente autorizada desde una óptica científica, la misma puede ser objetable con criterios exclusivamente técnico-científicos, siendo el caso que se contradice por el Informe también presentado por el Instituto Español de Oceanografía, el cual, no siendo tampoco la opinión "decisiva", sí es de singular relevancia en tanto "organismo de referencia" en la materia. Se contraponen, pues, dos dictámenes, y dos opiniones fundadas en consideraciones y apreciaciones científicas, y, aun reconociéndose por el Tribunal la incapacidad para "resolver" la controversia técnica, se concluye, al menos, sobre la insuficiencia, por existencia de contradicción, del informe inicial, con la desestimación de las pretensiones autonómicas. Como dice el TSJA, la determinación del concepto de "continuidad ecológica" entre el medio marino y el medio terrestre no resulta fácil, ni automática, por la propia definición y características de esta categoría jurídico-administrativa, por lo que hay que convenir, habiéndose pronunciado en contra el Instituto Español de Oceanografía, que no se ha logrado determinar, con evidencia científica suficiente, tal concepto, según la exigencia impuesta por el ordenamiento jurídico. Porque "no es la Administración del Estado la que ha de probar la incorrección de los informes aportados de adverso, sino que es la Comunidad Autónoma que pretende declarar y gestionar espacios naturales protegidos en el mar la que ha de acreditar dicha continuidad ecológica de un modo especialmente riguroso, toda vez que lo ha de acreditar mediante la mejor evidencia científica existente, lo que en el caso que nos ocupa no puede ser apreciado" (F. J. $\left.2^{\circ}\right)$. 


\section{Indefensión formal vs. indefensión material en los procedimientos de declaración de Zonas de Especial Conservación}

STSJA de 30 de mayo de 2017, rec. 447/2015

Según la STSJA (Sala de lo Contencioso-administrativo, Sección 3ạ, Sede de Granada) de 30 de mayo de 2017, rec. 447/2015, y recordando la precedente jurisprudencia restrictiva sobre la viabilidad invalidatoria de la ausencia procesal del trámite de audiencia, las situaciones de indefensión han de valorarse según las circunstancias de cada caso, sin emanar de la sola y simple infracción de las normas procedimentales, sino cuando la vulneración de las normas procesales lleva consigo la privación del derecho a la defensa, con perjuicio real y efectivo para los intereses afectados, no protegiéndose situaciones de simple indefensión formal, sino aquéllos supuestos de indefensión material, en los que se haya podido razonablemente causar un perjuicio al recurrente. En el caso planteado, el mero alegato limitado a la afirmación de que la indefensión se ha producido no es suficiente, concluye el TSJA, para estimarla concurrente: falta, así, la explicitación de las circunstancias y elementos del perjuicio real sufrido, esto es, de la limitación del derecho de defensa, poniendo de manifiesto cuáles eran las "alegaciones sobre su contenido en relación a las fincas de su pertenencia situadas dentro del perímetro declarado como Zona Especial de Conservación que no pudo hacer por habérsele imposibilitado tener conocimiento del proyecto".

\section{Daños por inundaciones y concurrencia pública de culpas}

STSJA de 4 de julio de 2017, rec. 991/2013

STSJA de 11 de julio de 2017, rec. 144/2013

STSJA de 11 de julio de 2017, rec. 1090/2013

Se plantea en las SSTSJA (Sala de lo Contencioso-Administrativo, Sección 1aㅡ, Sede de Granada) de 4 de julio de 2017, rec. 991/2013, de 11 de julio de 2017, rec. 144/2013 y de 11 de julio de 2017, rec. 1090/2013, la posible existencia de responsabilidad patrimonial de la Junta de Andalucía por su falta de diligencia en la evitación de la crecida de las aguas de la denominada Balsa del Sapo (La Mojonera, Almería). Por la parte recurrente se señala que, según el informe aportado, la causa fundamental de las inundaciones había sido la ausencia de 
un drenaje suficiente que permitiese un control de los niveles de las balsas en unas cotas de seguridad aceptables para las personas y bienes. La Junta de Andalucía se opone, por el contrario, aduciendo: falta de competencias específicas, ausencia de factores concurrentes causantes de la ruptura del nexo causal, como la posible responsabilidad del Ayuntamiento con competencias en materia de urbanismo, la existencia de un convenio de explotación del acuífero y, finalmente, la declaración de interés general de las obras relacionadas con el mismo, con atribución paralela de las competencias al Estado.

Estima el TSJA que la responsabilidad por los daños acaecidos en la finca de la parte demandante corresponde tanto a factores naturales como a la sobreexplotación de los recursos hídricos y a la falta de diligencia de la Administración General del Estado, que se había reservado expresamente las competencias sobre las obras de interés general -cuyo objeto y finalidad consistía, precisamente, en la reducción de la lámina de la balsa mediante su desagüe y acondicionamiento-, y no, por tanto, de la Administración autonómica, por el hecho de haber realizado obras de impulsión y bombeo, que en principio tendrían un carácter inequívocamente "accesorio o provisional", en tanto la Administración competente no concluyera las obras que le correspondían legalmente (F. J. 5º).

\section{La modificación de documentos gráficos urbanísticos no es en cualquier caso una mera rectificación de errores}

STSJA de 13 de julio de 2017, rec. 58/2013

La STSJA (Sala de lo Contencioso-Administrativo, Sección 4ª , Sede de Granada) de 13 de julio de 2017, rec. 58/2013, se enfrenta a un recurso interpuesto contra el Acuerdo del Pleno del Ayuntamiento de Cuevas de Almanzora de aprobación definitiva de la Ordenanza General Avance para la identificación y delimitación de los asentamientos existentes en suelo no urbanizable común/rural y hábitat rurales diseminados, así como de edificaciones aisladas en suelo no urbanizable común, por diversas irregularidades en su tramitación. Destaca dentro de los asuntos tratados uno concreto, interesante por su especificidad y por su propia relevancia desde el punto de vista de la garantía jurídica de incolumidad de la protección de 
determinados espacios. Se trata de la elaboración de un nuevo documento gráfico donde, a diferencia del anterior, se eliminan de la memoria y del plano de contenido sustantivo general de la ordenanza diversos ámbitos territoriales marcados como asentamientos, por diversos motivos (por comprobarse que se hallan en suelo urbano, por no contener los requisitos de la norma directora, etc.). La cuestión suscitada es si cabe aquí dicha modificación alegando mera rectificación de errores o se trata de una modificación sustancial encubierta o fraudulenta. $Y$ recuera el TSJA la actual doctrina restrictiva en torno a la funcionalidad de esta prerrogativa de rectificación de oficio de errores por parte de la Administración. No se cumplen, así, los requisitos jurisprudenciales exigidos, ya que no se trata de un mero error en la transcripción de planos, ni era posible apreciarlo teniendo en cuenta exclusivamente los datos del expediente administrativo, por lo que, realmente, se produce una alteración fundamental del sentido del acto (una revocación de oficio), incompatible con el reseñado criterio restrictivo que rige la aplicación de esta figura.

\section{Plazo de aprobación del deslinde del dominio público hidráulico y requisitos para su ampliación excepcional}

STSJA de 18 de julio de 2017, rec. 609/2013

Según la STSJA (Sala de lo Contencioso-Administrativo, Sección 1aㅡ, Sede de Granada) de 18 de julio de 2017, rec. 609/2013, la ampliación del plazo de resolución de estos expedientes de deslinde tiene carácter excepcional, exigiéndose, por ello, en primer lugar, una adecuada motivación sobre su necesidad real, sin que basten las meras alegaciones genéricas a la complejidad e imprevisibilidad de la duración de los trámites procesales necesarios, motivadas por las demoras en la práctica de las notificaciones personales a los interesados y de las publicaciones preceptivas, así como a las dificultades técnicas que entraña la determinación de los límites del dominio público hidráulico en la zona. Además, la ampliación del plazo no puede justificarse en circunstancias que ya existían cuando se inició el procedimiento, pues en ese momento ya sabía la Administración que tenía un plazo impuesto por ley de veinticuatro meses para notificar la resolución del procedimiento. Y, finalmente, tampoco se acredita que se hayan agotado realmente todos los medios a disposición posibles que justifiquen la excepcionalidad de la 
ampliación del plazo, sin que baste la mera afirmación (formal) al respecto. Se estima por ello el recurso y se acuerda, en consiguiente, la anulación de la resolución autonómica de aprobación del deslinde demanial cuestionado.

8. Virtualidad anulatoria de la ausencia de los informes técnicos y de suficiencia de recursos hídricos en los procedimientos de declaración de interés turístico de proyectos de campos de golf: declaración "por silencio" e inmanencia de "lesividad" de la contradicción con la normativa ambiental reguladora

\section{STSJA de 19 de julio de 2017, rec. 13/2016}

La STSJA (Sala de lo Contencioso-Administrativo, Sección 3aㅗ, Sede de Sevilla) de 19 de julio de 2017, rec. 13/2016, se pronuncia sobre la funcionalidad anulatoria o no de la ausencia de dos informes legalmente exigidos para la declaración de interés turístico de un campo de golf. En el primer caso se trataba del informe de la Comisión Técnica de Calificación, regulado como informe de carácter preceptivo y no vinculante, cuya omisión (y a la luz además del carácter desfavorable del informe extemporáneo) constituye causa de nulidad absoluta o de anulabilidad, al menos, pero en ningún caso de irregularidad no invalidante. En cuanto al segundo (informe de suficiencia de recursos hídricos) recuerda el TSJA su carácter "esencial" (por su trascendencia material) y "vinculante" (por su afección expresa al ámbito competencial de la Confederación Hidrográfica correspondiente), y, además, "previo" necesariamente a la declaración de interés turístico del proyecto, "pues la implantación del campo de golf sólo podrá llevase a cabo una vez garantizada la suficiencia y calidad hídrica".

Las características reseñadas de este segundo informe conducen a estimar su ausencia como causa de nulidad de pleno derecho, sin obviar además la necesidad de aportar autorización o concesión del organismo de cuenca correspondiente.

No obstante, lo que se interpone ante el TSJA es un recurso contenciosoadministrativo de lesividad contra la declaración de interés turístico, por lo que queda por resolver si efectivamente el acto declarado lesivo incide -o nonegativamente en el interés público, para legitimar tal acción de defensa. Es 
decir, se trataría de verificar la suficiencia argumentativa de las afecciones para el interés público sobre la normativa urbanística y de aguas derivadas de la declaración de interés turístico obtenida por "silencio positivo" (a pesar de la ausencia de los dos informes esenciales señalados). Y según el TSJS, tal "lesividad" concurre, puesto que son evidentes las razones urbanísticas y medioambientales afectadas por la declaración, con lo que las infracciones del ordenamiento vienen concretadas por la Administración en su resolución y su carácter lesivo para el interés público es "intrínseco" a dichas infracciones en materia de aguas y ordenación del territorio, de manera que si la Administración ha producido por silencio administrativo un acto que supone un perjuicio para el medio ambiente o la planificación municipal sin asiento normativo habilitante, y, por tanto, contra la normativa aplicable, ello, por su propia formulación, lleva ínsito el perjuicio para el interés público, y de ahí que la declaración de lesividad sea conforme a derecho (F. J. $4^{\circ}$ ). 